\title{
Kernos
}

Revue internationale et pluridisciplinaire de religion grecque antique

18 | 2005

Varia

\section{Jean-Baptiste BONNARD, Le complexe de Zeus. Représentations de la paternité en Grèce ancienne}

\section{Véronique Dasen}

\section{CpenEdition \\ Journals}

\section{Édition électronique}

URL : http://journals.openedition.org/kernos/1739

DOI : 10.4000/kernos. 1739

ISSN : 2034-7871

\section{Éditeur}

Centre international d'étude de la religion grecque antique

\section{Édition imprimée}

Date de publication : 1 janvier 2005

Pagination : $553-555$

ISSN : 0776-3824

Référence électronique

Véronique Dasen, « Jean-Baptiste BonNARD, Le complexe de Zeus. Représentations de la paternité en Grèce ancienne », Kernos [En ligne], 18 | 2005, mis en ligne le 24 mai 2011, consulté le 21 septembre 2020. URL : http://journals.openedition.org/kernos/1739; DOI : https://doi.org/10.4000/kernos.1739 
miel » réunit Platon, Pindare, le chrétien Ambroise, mais aussi Zeus lui-même dans une commune relation au miel. Rhésos, un « cousin d'Orphée », est mis en regard de son prestigieux parent par la vertu des rapprochements et des écarts de leur biographie respective. Avec "Écho, désir, musique, les sentiers de Pan», Ph. B. retrouve le chemin de ses Recherches sur le dieu Pan (1979) et rejoint La Mère des dieux (1996) sur le thème de la musique. Quant à «la mort du Grand Pan », ce chapitre offre une remarquable leçon de méthode autour du curieux épisode rapporté par Plutarque (De la disparition des oracles, 17). S'interrogeant à la fois sur la portée du récit en contexte antique et sur les acrobaties interprétatives qui se sont succédé entre $\mathrm{XIX}^{\mathrm{e}}$ et $\mathrm{Xx}^{\mathrm{e}}$ siècle, $\mathrm{Ph}$. B. montre que le récit relève d'une propagande politique anti-impériale, revue ensuite dans une perspective apologétique chrétienne. En outre - et la leçon de méthode est là -, le comparatisme totalisant qui l'a intégré dans l'imagerie du « dieu qui meurt » ou dans le cadre folklorique de légendes nordiques montre avant tout la distance qui sépare les données et la succession de modes interprétatives. Ensuite, «la tête du Capitole » est une analyse fine et comparative des mécanismes idéologiques et historiques ayant mené à cette tradition: mise au jour d'une tête sur la colline tarpéienne, exploration du sens du prodige, tentative de détournement de l'oracle, reconnaissance de l'hégémonie à venir de Rome.

Le dernier volet de l'ouvrage n'entre pas tout à fait dans « l'exercice mythologique» du titre, même s'il a pleinement sa place dans ce regard rétrospectif sur un parcours, à la fois scientifique et personnel. Ph. B. fut l'élève de Mircea Eliade à Chicago dans les années septante et cette relation, même si elle fut moins déterminante que celle de Jean Rudhardt, a marqué le chercheur débutant en histoire des religions. Ce chapitre final, intitulé «Un mythe moderne : Mircea Eliade », est une mise au point rigoureuse et sensible de l'impact des « années noires » du savant roumain, quand il adhérait aux idées extrémistes de la Garde de Fer de Codreanu (1937-1944). Elle est rigoureuse car elle rend compte des dérives auxquelles un nationalisme exalté, soucieux de « respiritualiser l'individu», a mené Eliade, tout en continuant d'informer dans une certaine mesure sa vision méta-historique du sacré. Elle est sensible car certains excès du «révisionnisme» auquel a été soumise la carrière d'Eliade après sa mort ont manifestement blessé son ancien élève, qui a connu un autre homme "qu'il serait vain, absurde de vouloir à tout prix réduire au premier » (p. 202).

Ce livre remarquablement écrit montre la cohérence d'une démarche et l'application d'une méthode d'analyse des mythes qui rend compte de leurpoikilia en leur appliquant, sans exclusive, les acquis les plus intéressants des trois dernières décennies.

$$
\begin{aligned}
& \text { Vinciane Pirenne-Delforge } \\
& \text { (FNRS - Université de Liège) }
\end{aligned}
$$

Jean-Baptiste Bonnard, Le complexe de Zeus. Représentations de la paternité en Grèce ancienne, Paris, Publications de la Sorbonne, 2004. 1 vol. $16 \times 23,5$ cm, 254 p. ISBN : 2-85944-508-0.

Le discours grec sur la place du père dans le processus de la génération est au coeur de cet ouvrage tiré d'une thèse sur «La représentation du père de la cité. Contribution à l'étude de l'imaginaire dans la Grèce archaïque et classique », soutenue en 1998 à Paris I Panthéon-Sorbonne. J.-B. Bonnard y compare deux dossiers, l'un mythique, l'autre biologique, pour analyser les différentes manipulations discursives qui traduisent l'idéal d'un monde masculin déniant aux femmes le pouvoir de la reproduction. La démarche est originale et s'inscrit dans un courant comparatif qui 
s'attache à confronter différents niveaux de représentation qui mettent en œuvre, chacun à sa manière, l'imaginaire collectif d'une société. Sur le plan mythique, comme dans le discours scientifique, la pensée grecque privilégie ainsi le rêve d'un monde où la reproduction de l'humanité se passe des femmes ou les relègue à une place mineure.

L'ouvrage est divisé en deux parties. La première examine les facettes de la fonction génératrice du père dans un discours mythique qui décrit des paternités solitaires exemplaires, la naissance de héros sans mère, et raconte des paternités humaines parodiques. Parmi les dieux, Zeus est le champion des naissances monoparentales (Athéna, Dionysos, Agdistis et Aphrodite), avec Ouranos, dont le sang du sexe castré produit de nombreuses divinités, tels les Géants, les Érinyes, les Nymphes. La survalorisation du rôle paternel se traduit par la naissance de jumeaux issus d'une double paternité. À côté des Dioscures et d'Héraclès et Iphiclès, conçus par Zeus associé à Amphitryon ou à Tyndare, plusieurs autres paires gémellaires pourraient être évoquées pour renforcer la démonstration de l'auteur ( $c f$. V. Dasen, Jumeaux, jumelles dans l'Antiquité grecque et romaine, Akanthus Verlag, 2005). Les descriptions mettent l'accent sur les douleurs de l'enfantement masculin et nous rappellent que les souffrances sont la part virile de la fonction maternelle qui se voit reconnaitre grâce à elles une certaine andreia. On retrouve l'expression d'un désir de paternité exclusive dans les mythes de l'autochtonie béotienne et athénienne. Cadmos, «semeur des Spartes », et Érichthonios, "enfant sans mère », fondent un ordre politique dont les femmes sont exclues. Les élucubrations de Lucien sur les engendrements masculins des Séléniens, qui naissent dans les mollets, ou des Dendrites, issus d'un testicule mis en terre, brodent sur le même fantasme. Quand au corps maternel, la métaphore très répandue du sillon le dévalorise en le réduisant à une fonction nourricière. À ce traitement du corps féminin, transformé par analogie en paysage, on pourrait ajouter la dimension de la mère comme territoire, bien explorée par Susan Guettel Cole (Landscapes, Gender and Ritual Space, 2004).

La deuxième partie de l'ouvrage livre le dossier du mécanisme de la génération humaine dans le discours biologique, des Présocratiques à Aristote en passant par le corpus hippocratique. L'idée de l'existence d'une semence féminine à côté d'une semence masculine ne devient générale qu'au $\mathrm{v}^{\mathrm{e}}$ siècle, mais sans que leurs rôles respectifs ne soient jugés véritablement égaux. Pour les Hippocratiques, chaque sexe est porteur d'une semence mâle et femelle, mais l'élément femelle est dévalorisé par un ensemble de connotations organisées selon un système d'oppositions binaires (froid/chaud, humide/sec, gauche/droite). L'écart se creuse encore chez Aristote qui oppose le sang menstruel, semence impure mais nourricière, au sperme masculin, porteur du souffle créateur, vital. La femme n'est qu'un mal nécessaire pour perpétuer l'espèce, et exclue de toute participation à la détermination du sexe ou des caractères de l'enfant.

L'argumentation de chacune des deux parties s'appuie sur un riche dossier de textes cités en grec et accompagnés d'une traduction. L'ouvrage, assorti d'une utile bibliographie et d'indices (mythologicum, fontium, nominum), constitue une contribution bienvenue à l'histoire des pères et de la paternité, encore paradoxalement peu explorée. Il ouvre aussi plusieurs champs de recherches prometteurs, par exemple pour une remise en question du modèle du corps unisexe théorisé par Thomas Laqueur (La fabrique du sexe. Essai sur le corps et le genre en Occident, 1992). Si le concept du corps unisexué peut s'appliquer à la construction aristotélicienne, le modèle hippocratique y résiste. La valorisation du masculin sur le féminin y est 
indéniable, mais les auteurs y définissent deux types de chair, féminine et masculine, dont la différence est radicalisée par la présence de l'utérus, organe féminin - et maternel - par excellence.

Véronique Dasen

(Université de Fribourg)

Françoise Frontisi-Ducroux, L’homme-cerf et la femme-araignée, Paris, Gallimard, 2003. 1 vol. $16,5 \times 21$ cm, 300 p., 70 ill. (Le Temps des images). ISBN : 2-07-076655-1.

Ce livre important nous mène au cour du fonctionnement de l'imaginaire collectif grec au travers des déclinaisons presque infinies des métamorphies humaines et divines dans la mythologie classique. Face à la profusion des récits, Fr. FrontisiDucroux s'est centrée sur les histoires qui font l'objet de représentations figurées, sans négliger les récits parallèles dépourvus de version iconographique ou qui ne sont connus que par les images. Avec beaucoup de finesse, l'A. fait patiemment émerger de la confrontation du système verbal avec les procédés de réthorique picturale les spécificités des catégories mentales et symboliques grecques. Les principaux axes de réflexion s'articulent autour de la perméabilité des frontières entre l'humain, l'animal, le végétal et le minéral, traduite par différentes formes d'hybridation, parfois incomplètes ou doublées d'un changement de sexe, autour des rapports entre la féminité et l'animalité, le désir et la bestialité, enfin de la métamorphose comme expression particulière d'une temporalité perçue non comme un continuum mais comme une succession de stades distincts.

Les récits examinés sont essentiellement livrés par des auteurs grecs, mais sans faire l'économie d'Ovide, qui le premier utilise le mot grec Metamorphoseon dans le titre de son poème Les métamorphoses. Conservatoire de récits disparus, son recueil est précieux à plus d'un titre, notamment pour sa part de réélaboration qui permet d'approcher d'autres façons culturelles de penser le corps et l'hybridation.

Le livre est découpé en six chapitres qui tournent chacun autour d'un mythe principal et d'une thématique. Le premier chapitre explore l'hybridité et la polymorphie des créatures qui hantent les mers, tels Nérée, sa fille Thétis, Protée, Glaucos et Métis. Leur métamorphie n'est pas la conséquence d'un accident ni d'un acte volontaire, mais inhérente à leur nature, changeante et fluide comme l'eau. Leur passage d'une forme à l'autre est traduite visuellement par des formes composites juxtaposant des appendices animaux (poisson, lion, panthère, chèvre, serpent). L'épisode de la lutte de Thétis et Pélée détient une valeur paradigmatique. Il rappelle la fonction profonde du mariage, maîtriser la nature féminine en fixant son instabilité foncière. - Le deuxième chapitre explore autour du mythe de Circé les limites d'une bestialisation qui ne prive pas de conscience humaine les hommes enfermés dans une enveloppe animale. Les imagiers inventent des assemblages composites, greffant des parties animales sur des parties humaines, usant de métonymie visuelle pour rendre visible la transformation des victimes. L'emploi constant de l'aoriste pour décrire le phénomène traduit l'impossible représentation du temps de la métamorphose, insaississable pour les mortels; la valeur ponctuelle de l'action verbale renvoie à l'instantanéité de l'action divine. - Le troisième chapitre interroge le statut de la vision par le biais du mythe d'Actéon qui bascule dans l'animalité à cause de son voyeurisme. Chasseur devenu proie, il est dévoré par ses chiens. Sa métamorphose en cerf est-elle réelle ou les chiens sont-ils sujets d'une hallucination? Le sort d'Actéon renvoie à celui de Tirésias qui change de sexe en punition de son regard transgressif. 